\title{
Automated segmentation of the corpus callosum in midsagittal brain magnetic resonance images
}

\author{
Chulhee Lee, MEMBER SPIE \\ Shin Huh \\ Yonsei University \\ Department of Electronic Engineering \\ 134 Shinchon-Dong, Seodaemoon-Gu \\ Seoul 120-749, Korea \\ E-mail: chulhee@yonsei.ac.kr
}

Terence A. Ketter

Stanford University School of Medicine

Department of Psychiatry and Behavioral Sciences

Stanford, California 94305-5723

Michael Unser, MEMBER SPIE

Swiss Federal Institute of Technology

EPFL, DMT/IOA-Biomedical Imaging Group

P.O. Box 127

1015 Lausanne, Switzerland

\begin{abstract}
We propose a new algorithm to find the corpus callosum automatically from midsagittal brain MR (magnetic resonance) images using the statistical characteristics and shape information of the corpus callosum. We first extract regions satisfying the statistical characteristics (gray level distributions) of the corpus callosum that have relatively high intensity values. Then we try to find a region matching the shape information of the corpus callosum. In order to match the shape information, we propose a new directed window region growing algorithm instead of using conventional contour matching. An innovative feature of the algorithm is that we adaptively relax the statistical requirement until we find a region matching the shape information. After the initial segmentation, a directed border path pruning algorithm is proposed in order to remove some undesired artifacts, especially on the top of the corpus callosum. The proposed algorithm was applied to over 120 images and provided promising results. () 2000 Society of Photo-Optical Instrumentation Engineers. [S0091-3286(00)00604-8]
\end{abstract}

Subject terms: medical imaging; image segmentation; corpus callosum; magnetic resonance images; region matching; directed window region growing.

Paper 990146 received Apr. 2, 1999; revised manuscript received Sep. 8, 1999; accepted for publication Sep. 13, 1999.

\section{Introduction}

Object recognition is often one of the ultimate goals in image processing, computer vision, and artificial intelligence (AI). Although fully automated general object recognition is far beyond the reach of current technology, specialized object recognition can be very useful in many applications. Object recognition typically involves two distinct processing steps: (a) finding boundaries and (b) matching. Each of these aspects of the problem has been an important research area on its own. Finding boundaries between regions (segmentation) is the first step for various analyses in image processing. There are several review papers on segmentation, ${ }^{1-3}$ including thresholding, ${ }^{4,5}$ edge detection, ${ }^{6}$ spatial interaction models such as the Markov Random Field (MRF) and the Gibbs Random Field (GRF), and neural networks. ${ }^{7}$ However, due to the complex nature of images, no algorithm is consistently able to find good boundaries across various types of images. Since shape matching depends on finding good boundaries, this can cause serious problems in object recognition. In particular, if an object is non-rigid and part of the boundary is missing, which is typical in medical images, algorithms based on contour matching may not work well. Active contour models, also known as snakes, have been widely used in medical images to find certain objects. ${ }^{8,9}$ Snakes, however, have one important limitation: they require good starting conditions. They are therefore primarily useful as an interactive contour outlining tool and not really suitable for the fully automated detection of complex structures such as the corpus callosum.
Segmentation and object recognition have been of a great interest in medical imaging. ${ }^{10-14}$ Related work includes a segmentation method using game theory, ${ }^{15}$ segmentation using objective functions, ${ }^{16} 3$-D segmentation of brain images, ${ }^{17}$ detection of blood vessels in retinal images, ${ }^{18}$ tumor detection, ${ }^{19}$ segmentation and object recognition in echocardiograms, ${ }^{20,21}$ and segmentation of cardiac MR images. ${ }^{22}$

The corpus callosum, which is located at the center of the brain (Fig. 1), is the major communication pathway between the two cerebral hemispheres and mainly consists of axons. Structural changes in the corpus callosum occur in a variety of neurological diseases. ${ }^{23}$ Also, a substantial number of studies have investigated the corpus callosum in schizophrenia ${ }^{24-33}$ with a meta-analysis of 11 studies suggesting that corpus callosum area (but not length or corpus callosum/brain ratio) in midsagittal images was decreased in schizophrenia patients compared to healthy controls. ${ }^{34}$ In addition, some, ${ }^{35,36}$ but not all ${ }^{31,37}$ studies in mood disorders have reported changes in the corpus callosum. In all cases, the corpus callosum was separated from surrounding tissues manually or interactively with aid of computer graphics. This is a time consuming process and the results may be influenced by subjective bias. Thus, an automated objective method to find the corpus callosum will greatly facilitate such studies.

The corpus callosum is also an important landmark in midsagittal MR brain images. It lies largely inferior to the cerebrum, superior to the brainstem, and antero-superior to the cerebellum. Thus, finding the corpus callosum auto- 
(a)

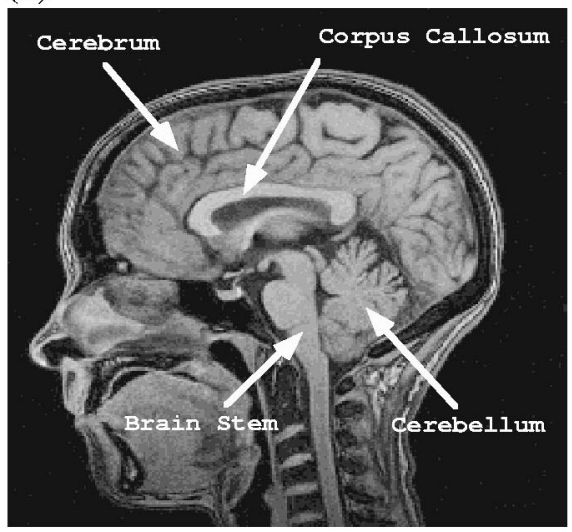

(b)

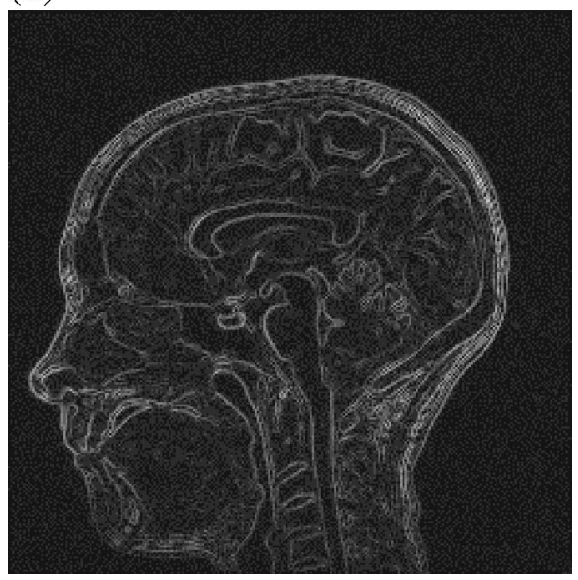

Fig. 1 Corpus callosum (a) in a midsagittal brain MR image and its edge (b).

matically would be helpful in providing valuable reference points to locate other brain structures and applications such as accurate image registration among individuals and automatic segmentation of the brain. Therefore, if the corpus callosum can be extracted automatically, it can be used for medical diagnosis, accurate image registration, and automatic segmentation. However, the boundaries between the corpus callosum and surrounding tissues are not always clear and variations of size and shape among individuals are significant.

In this paper, we investigate the problem of finding the corpus callosum automatically in magnetic resonance images (MRI) and propose a robust region-based solution that consists of a series of operations. Instead of finding boundaries and matching contour, ${ }^{38}$ the proposed algorithm is based on region information. Initially, we extract the statistical characteristics of the corpus callosum and obtain global shape information. In order to locate the object of interest, we first select regions that meet the statistical criteria and then find the one that matches the shape information. Finally, in order to remove regions that tend to be incorrectly merged to the top of the corpus callosum, we propose border path pruning that successfully removes the artifacts.

\section{Statistical Characteristics and Shape of Corpus Callosum}

Fig. 1a shows a typical midsagittal brain MR image and Fig. $1 \mathrm{~b}$ shows edges extracted from Fig. 1a by applying a (a)

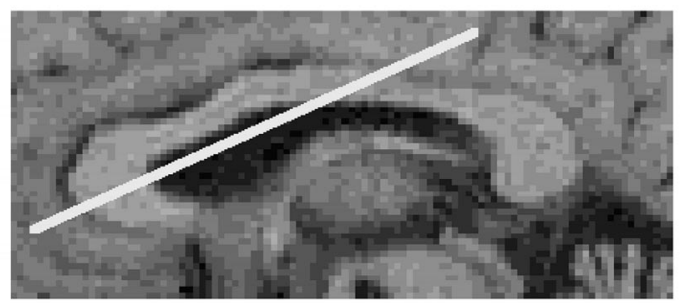

(b)

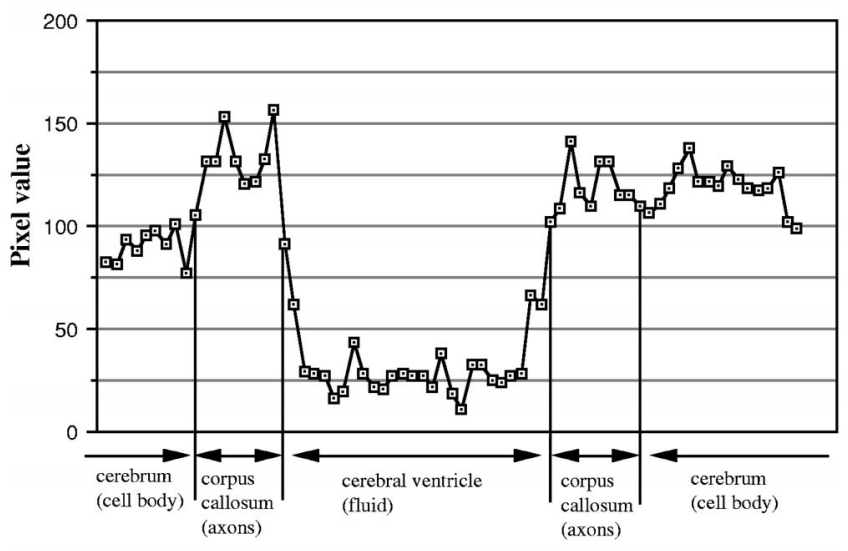

Fig. 2 A cross-section of the corpus callosum.

gradient operation. Although one can visually recognize the outline of the corpus callosum in Fig. 1b, portions of its boundary are indistinct, which can make it difficult to develop an automated recognition algorithm based on edge information alone. A problem with applying edge detection algorithms is that, quite often, variation within the corpus callosum can be comparable or exceed the difference between the corpus callosum and surrounding tissues. In addition, parts of the boundary between the corpus callosum and surrounding tissues are indistinct and thus very difficult to define due to similar gray levels, particularly on the top portion of the corpus callosum and between the corpus callosum and the fornix. These problems are illustrated in Fig. 2. Fig. $2 b$ shows the gray level along the cross line of Fig. 2a. As can be seen, the variation in the corpus callosum is as large as the difference between the corpus callosum and the cerebrum.

Fig. 3 shows pixel value histograms of the corpus callosum and whole brain derived from an MR image. The number of gray levels of all the images used in this paper is 256. It can be seen that the corpus callosum has relatively high intensity values. Therefore, if we apply a threshold starting at a high value, the corpus callosum will generally begin to appear before other brain structures show up in the binary image. Furthermore, with such thresholding, few other structures will be present in the thresholded binary image (Fig. 4), which will make the corpus callosum recognition algorithm much more efficient and robust. Fig. 4 shows binary images obtained by applying decreasing thresholds. With $t=150$, we can barely recognize the corpus callosum. With $t=130$, a relatively distinct callosal shape is evident with few other non-adjacent structures. 


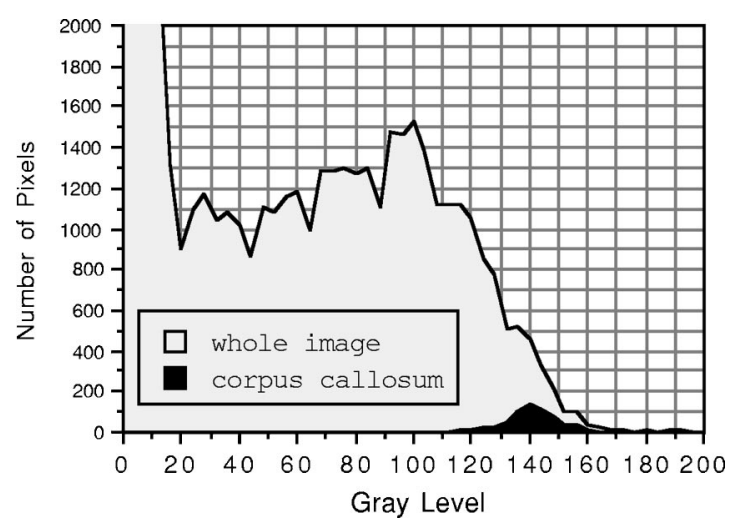

Fig. 3 Histograms of the corpus callosum and the brain MR image.

With $t=120$, the corpus callosum is clearly defined, although more other non-adjacent structures are visible. With $t=110$, the corpus callosum starts to be connected to surrounding tissues. Although the threshold values may be different depending on individual images, this property of high intensity values of the corpus callosum can be ex-

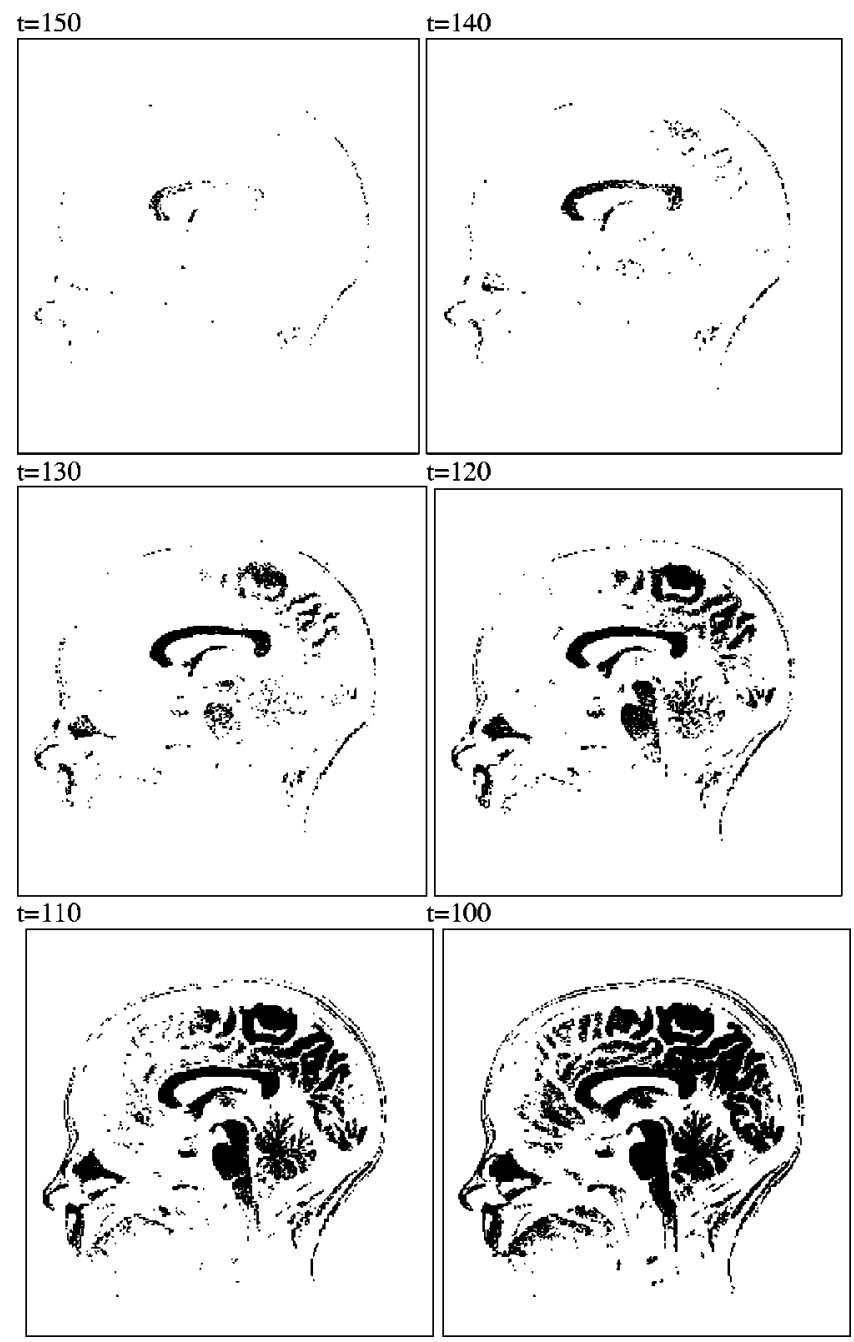

Fig. 4 Thresholding with various threshold values.
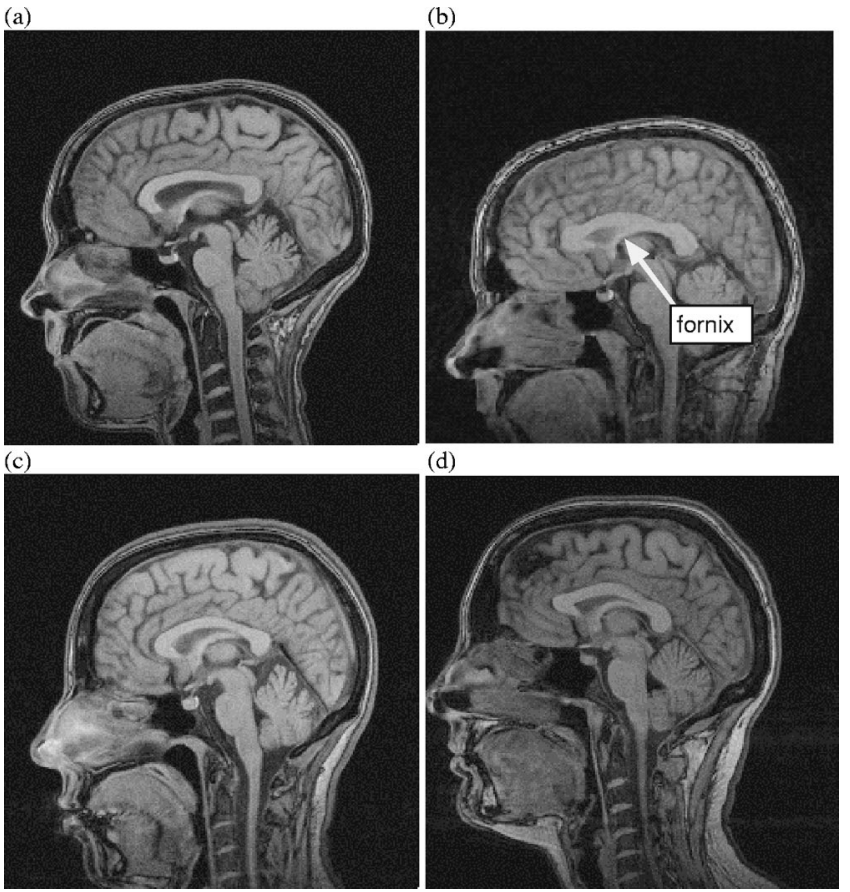

(d)

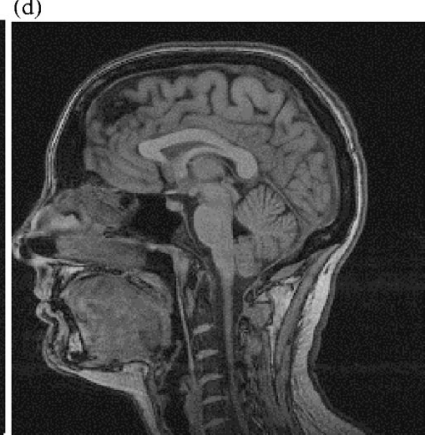

Fig. 5 Size and shape variations of corpus callosum.

ploited to yield a segmentation algorithm that is efficient across images.

Fig. 5 illustrates the substantial variation in size, shape, location, and orientation of the corpus callosum across individuals. In particular, the shape is difficult to define because the fornix, a tail-shaped structure with similar composition (axons) anteriorly descending from mid to posterior corpus callosum, is hard to distinguish from the corpus callosum, as can be seen in Fig. 5b and Fig. 5d. In many cases, the fornix has almost identical gray levels as the corpus callosum. As a result, it is very difficult to separate the fornix from the corpus callosum based on edges or gray level. This can pose a serious problem to contour matching. Although it is difficult to describe the shape of the corpus callosum exactly, it can be generally said that it is arc-shaped and that its length is about one third of that of the skull.

In order to obtain size statistics of the corpus callosum, we semi-manually extracted the corpus callosum from 20 adult subjects; Table 1 provides size statistics estimated from the 20 adult subjects. We assume that in adults the minimum length is 55 pixels ( 10 pixels less than the minimum and more than 3 standard deviations less than the mean in our sample) and the minimum height is 18 pixels (5 pixels less than the minimum and more than 3 standard deviations less than the mean in our samples).

Table 1 Size of the corpus callosum in pixels (MR image size is 256 by 256), based on 20 adult subjects.

\begin{tabular}{ccccc}
\hline \hline & Mean & sd & Max & Min \\
\hline Length & 73.1 & 4.9 & 85 & 65 \\
Height & 27.2 & 2.9 & 33 & 23 \\
\hline \hline
\end{tabular}


Lee et al.: Automated segmentation of the corpus callosum in midsagittal . . .

Table 2 The size, length, height of the corpus callosum for various thresholds.

\begin{tabular}{cccc}
\hline \hline Threshold & Area (pixels) & Length & Height \\
\hline 99 & 295 & 53 & 35 \\
98 & 313 & 54 & 35 \\
97 & 326 & 54 & 35 \\
96 & 337 & 54 & 35 \\
95 & 512 & 84 & 35 \\
94 & 532 & 84 & 35 \\
93 & 556 & 84 & 36 \\
92 & 559 & 84 & 36 \\
91 & 565 & 84 & 36 \\
90 & 570 & 84 & 36 \\
89 & 577 & 84 & 36 \\
88 & 581 & 84 & 36 \\
87 & 589 & 84 & 36 \\
86 & 678 & 96 & 39 \\
\hline \hline
\end{tabular}

Table 2 shows the area, length, and height of the corpus callosum for various thresholds for an individual midsagittal MR image. As the threshold decreases, the area (number of pixels), length and height of the corpus callosum increase as expected. It is observed that there are area and length discontinuities between $T=96$ and $T=95$. At this threshold, it meets the minimum length and height requirements. There are also area, length, and height discontinuities between 87 and 86 . Starting at this threshold, surrounding tissues begin to be connected to the corpus callosum. From the table, the optimal threshold appears to be between $T=95$ and $T=87$ for this image. Within this range, although the length and the height are virtually the same, there is a substantial variation in the area. A reason for the area difference is that for high threshold values, small holes were observed inside the corpus callosum, which need be filled with some post-processing such as morphological operations. When holes inside the corpus callosum are filled, the difference becomes minor. Thus, we choose the smallest threshold, because it provides the most complete structure.

Our detection strategy is to apply a threshold to the image starting with a high value and find a region that matches the shape information of the corpus callosum. An advantage of working on binary images is that the matching will be much easier since there are fewer other structures at high threshold values. In order to determine whether an object in the binary image matches the shape information of the corpus callosum, we propose the directed window region growing algorithm that is described in the next section.

\section{Directed Window Region Growing and the Recognition Algorithm}

In the proposed directed window region growing algorithm, we restrict the direction of region growing. For example, in Fig. 6a, region growing can only proceed from left to right (anterior to posterior in MR images). If we have information about the horizontal length of an object, we can find it with the directed window region growing algorithm. Fig. (b)
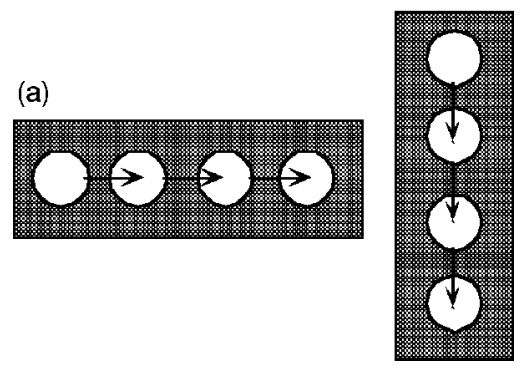

Fig. 6 Directed window region growing, (a) horizontal object, (b) vertical object.

$6 \mathrm{~b}$ illustrates another case where region growing can only proceed from top to bottom (superior to inferior in MR images). It is noted that we do not rely on contours to find objects. Since contours are not always well defined and portions may be missing, this can be an important advantage. By restricting horizontal and vertical directions together, we can follow more general shapes as shown in Fig. 7. Furthermore, in the directed window region growing, we use a window to reduce noise effects since it is more reliable to use the center of the largest circles on the next vertical line in order to compute the angle. The size of this window changes with the thickness of the corpus callosum, and we use circular regions.

In order to find the shape of the corpus callosum using the directed window region growing algorithm, we first find the largest circle that includes the leftmost (most anterior) point of the candidate region as shown in Fig. 7. Let $P_{1}$ be the center of the circle. The center of the next circular region to the right (posterior), $P_{2}$, is defined to be on a vertical line 10 pixels rightwards (posterior) from $P_{1}$. And the angle that line $\overline{P_{1} P_{2}}$ makes with the horizontal line must be between $20^{\circ}$ and $70^{\circ}$. The values of these and the following angles were estimated from sample images. The center of the next circular region to the right (posterior), $P_{3}$, is similarly defined to be on a vertical line 10 pixels rightwards (posterior) from $P_{2}$. The angle that line $\overline{P_{2} P_{3}}$ makes with line $\overline{P_{1} P_{2}}$ must be between $+20^{\circ}$ and $-50^{\circ}$, based on the manual examination of sample images (Table

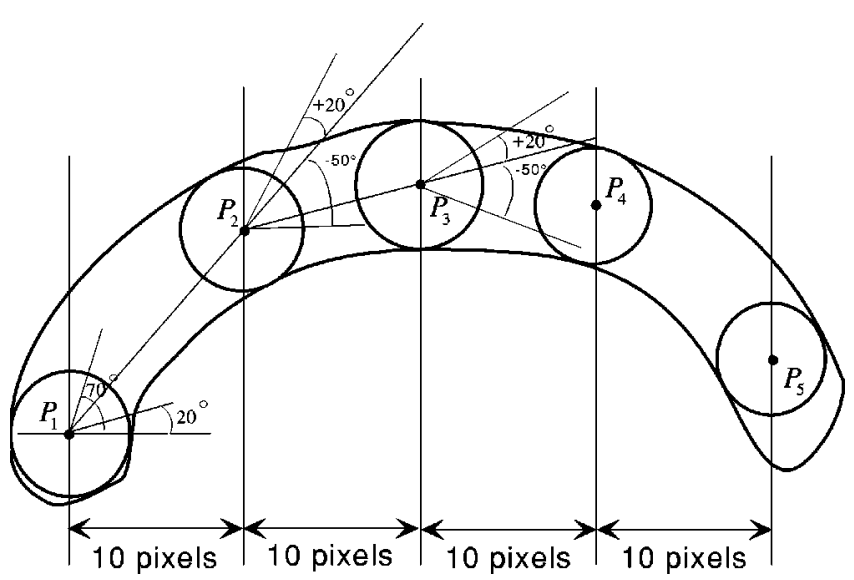

Fig. 7 Directed window region growing for a general shape. 
Table 3 Angle statistics obtained from sample images. $A 1$ is the angle that line $\overline{P_{1} P_{2}}$ makes with the horizontal line. $A 2$ is the angle that line $\overline{P_{2} P_{3}}$ makes with line $\overline{P_{1} P_{2}}$, and so on.

\begin{tabular}{crrrrrr}
\hline \hline & $A 1$ & \multicolumn{1}{c}{$A 2$} & \multicolumn{1}{c}{$A 3$} & \multicolumn{1}{c}{$A 4$} & \multicolumn{1}{c}{$A 5$} & \multicolumn{1}{c}{$A 6$} \\
\hline Max & 54.46 & -10.01 & 5.10 & -5.39 & 11.42 & 4.76 \\
Min & 30.96 & -39.29 & -33.40 & -33.40 & -22.41 & -36.67 \\
Ave & 41.87 & -20.16 & -14.74 & -18.85 & -5.08 & -11.03 \\
sd & 5.26 & 6.44 & 9.52 & 8.12 & 9.57 & 12.17 \\
\hline \hline
\end{tabular}

3). The centers of the remaining circular regions are obtained in a similar manner, proceeding from left to right (anterior to posterior) until there are less than 10 pixels within the candidate region to the right (posterior) of the center of a circle. Finally, if $P_{2}$ is above $P_{1}$ and the center of the last circular region $\left(P_{5}\right.$ in Fig. 7$)$ is below that of the previous region ( $P_{4}$ in Fig. 7), it can be said that the object has the shape of upward arc.

We thus summarize the characteristics of the corpus callosum as follows: Its gray level is among the highest in the brain (Fig. 3). In general, its minimum length is 55 pixels and minimum height is 18 pixels in a 256 by 256 pixel image (Table 1). Although its shape varies significantly between individuals, it is usually in the form of upward arc (Fig. 5), a condition that can be enforced using the directed window region growing method. Based on these observations, we propose the following procedure to segment the corpus callosum automatically:

\subsection{Procedure for Finding Corpus Callosum}

1. Set the initial threshold $t=t_{0}$.

2. Apply the threshold to the image.

3. Apply the directed region growing algorithm to the binary image. If there is a region that matches the shape description (upward arc, minimum length $=55$, minimum height $=18$ ), we have found the corpus callosum. Otherwise, decrease the threshold by 1 and go to Step 2.

\section{Post-Processing}

\subsection{Border Path Pruning}

In some cases, we face the problem that the corpus callosum and surrounding structures are joined together and therefore difficult to separate using gray level information. This happens especially with the cingulate cortex superior to the corpus callosum (cf. Fig. 8). This region may be

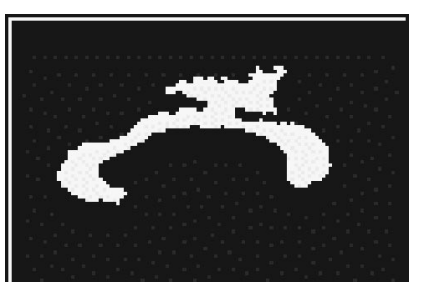

Fig. 8 Corpus callosum with artifact.

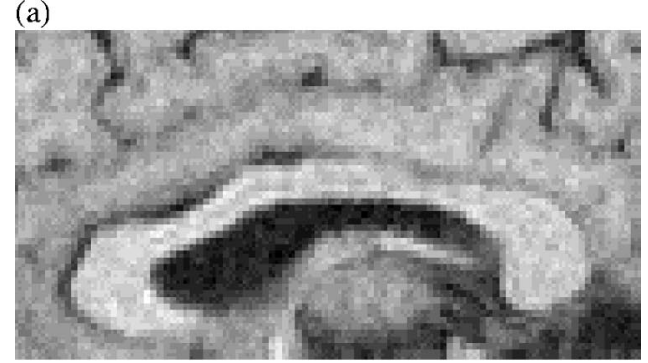

(b)
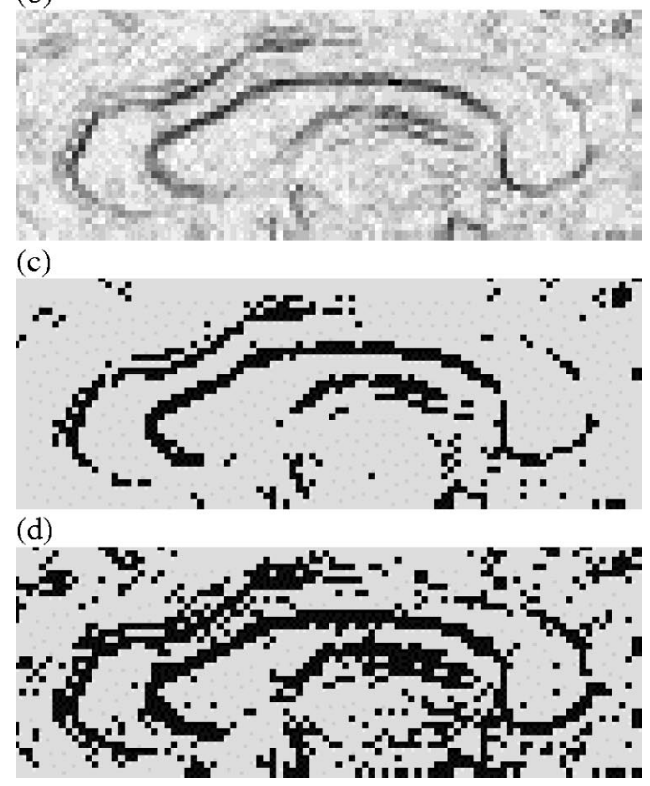

Fig. 9 (a) the original image, (b) the image obtained by applying a gradient operation to (a), (c) the binary image obtained by applying a threshold ( $T=30$ ) to (b), (d) the binary image obtained by applying a threshold $(T=20)$ to $(b)$

attached to the corpus callosum as the boundary may be indistinct. This is illustrated in Fig. 9 which shows an enlarged view of a corpus callosum; part of the boundary is indistinct and essentially non-detectable using conventional contour detection methods. Due to the nature of brain anatomy, this kind of problem can occur commonly in midsagittal brain MR images. In order to remove such undesirable artifacts, we apply the post-processing method described below.

First, we define a border in a binary image. A border consists of two adjacent pixels. One of the pixels belongs to the object and the other to the background. Depending on orientation, there can be a horizontal border or a vertical border, as illustrated in Fig. 10. A border path is defined as

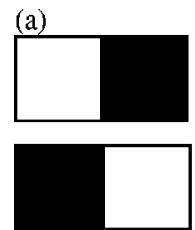

(b)

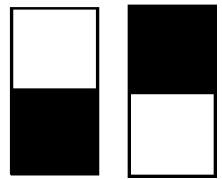

Fig. 10 Examples of the border consisting of two adjacent pixels. One of the pixels belongs to the object and the other to the back ground: (a) horizontal border, (b) vertical border. 


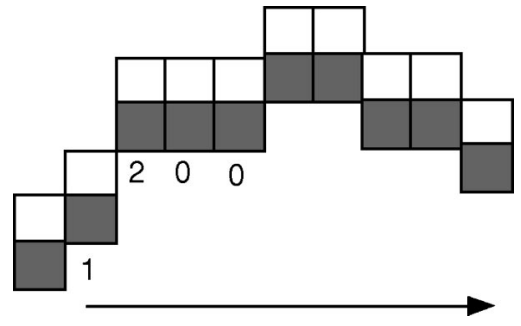

Fig. 11 An example of horizontal border path that is a sequence of vertical borders.

a sequence of borders, as shown in Fig. 11. Depending on orientation, there are two kinds of border paths: horizontal border path and vertical border path. Since artifacts tend to occur superior to the corpus callosum, we will use the horizontal border path to remove unwanted regions attached to the corpus callosum. We call this method border path pruning. In this procedure, we restrict the direction and maximum discontinuity of the border path. Fig. 12 illustrates border path pruning. Assuming that we require that border path propagation is uni-directional and has a maximum discontinuity of 2 pixels, there is no border path in Fig. 12a since the path violates the maximum discontinuity restriction. In Fig. 12b, there is no border path since the path violates the uni-directional restriction.

We propose using border path pruning to remove artifacts attached on the top of the corpus callosum and to determine whether the fornix is attached or not. From the equally spaced points used in the region growing algorithm, we draw vertical lines and find border points. For the detected shape to be acceptable, we require that there exists an uni-directional border path between any two of these adjacent border points. In the case of Fig. 13a, there exists one border path in every interval. However, in Fig. 13b, there is one interval where no border path exists. Usually, if there is an artifact, there is no border path. In that case, we draw a box containing the problematic interval and refine the threshold within this box (Fig. 13c). Specifically, we increase the threshold until we find a border path within this box. Although the corpus callosum can be barely distinguishable from the surrounding tissues, often there is a boundary whose gray level is slightly lower than that of the (a)

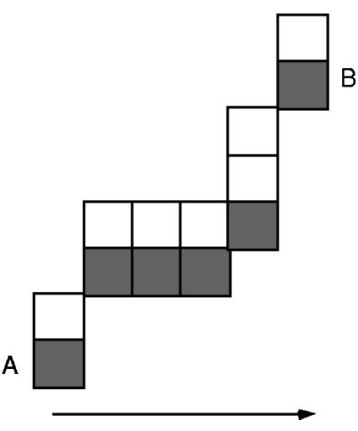

(b)

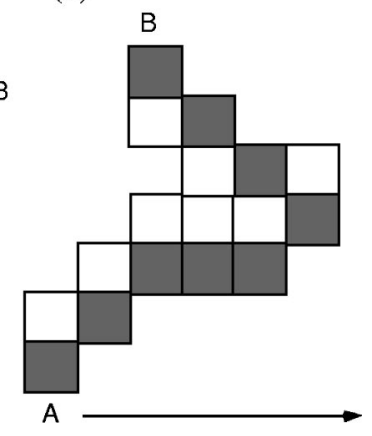

Fig. 12 Examples of border path pruning. (a) There is no border path since the path violates the maximum discontinuity restriction $(\leqslant 2)$. (b) There is no border path since the path violates the unidirectional restriction. (a)

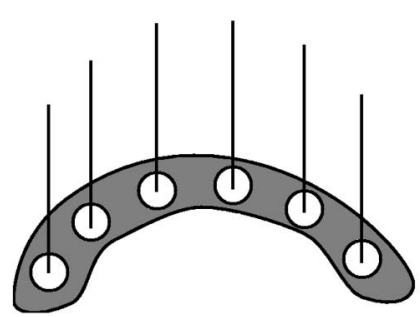

(b)

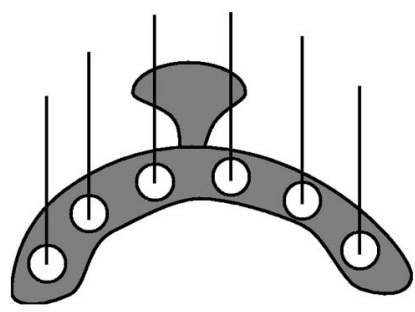

(c)

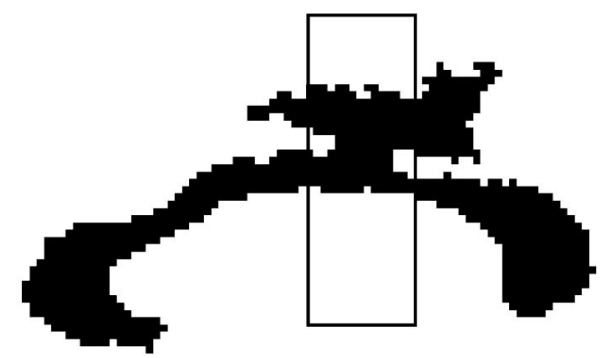

Fig. 13 Border path pruning. (a) There exists one border path in every interval. (b) There is an interval where no border path exists. (c) In order to remove artifacts, a box is drawn.

corpus callosum. Thus, border path pruning can separate the adjacent artifact from the corpus callosum in most cases. Fig. 14 shows the final result after the border path pruning. As can be seen, the artifact is successfully removed, though a part of the corpus callosum where the artifact was attached may be also lost in the process. However, we can restore some of the lost part by restoring the old area while keeping the new boundary. Similarly, by applying the border path to the inferior aspect of the corpus callosum, we can determine whether the fornix is attached to the corpus callosum and to locate the left (anterior) end point $A$ (Fig. 15), which will be described next.

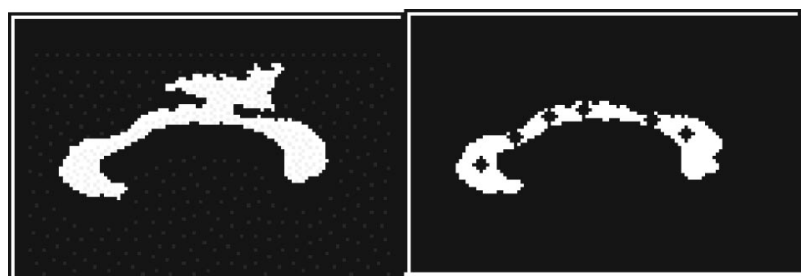

Fig. 14 Result of the border path pruning. The holes are just displayed to show where the centers of the circles are located. In the final images, there will be no holes. 


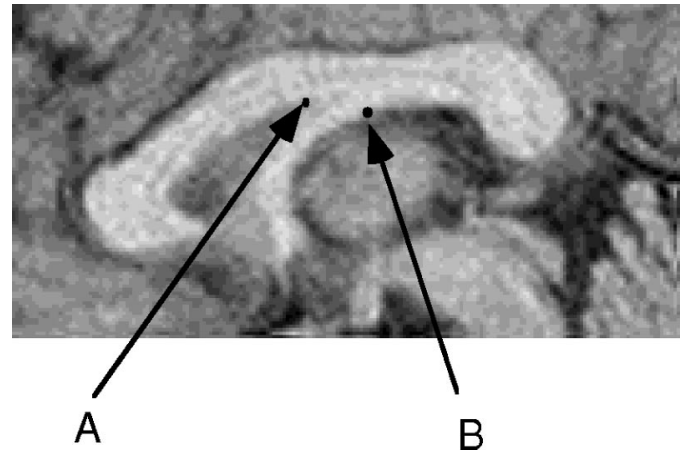

Fig. 15 Indistinct border between fornix and corpus callosum.

\subsection{Removing Fornix}

In some cases, the fornix is connected to the corpus callosum. The fornix appears as a tail-like protrusion descending anteriorly from the inferior aspect of the mid to posterior corpus callosum. Quite often, it has almost the same gray level as the corpus callosum and is very difficult to separate based solely on gray levels. Fig. 15 shows such an example. There are two points $(A$ and $B$ ) where the fornix meets the corpus callosum. We can apply the border path procedure to find the left (anterior) end point $A$. However, it may be more difficult to find the right (posterior) end point $B$. In some cases, additional information may be required. A possible solution would be extending a line posteriorly from $A$ which is parallel to the superior aspect of the corpus callosum until it hits a background region ( 0 in the binary image). We also may use, as a mask, the results of the parasagittal slices, which often lack fornix but still have corpus callosum. The methods provided reasonable performance but had some limitations. Fig. 16 shows some of the
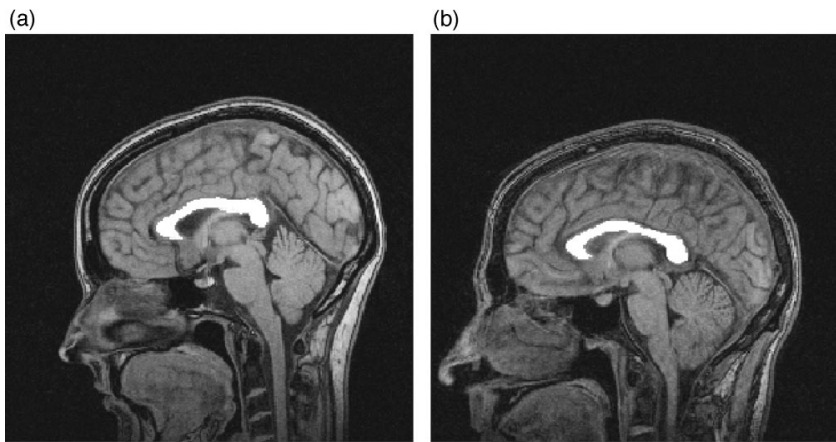

(c)
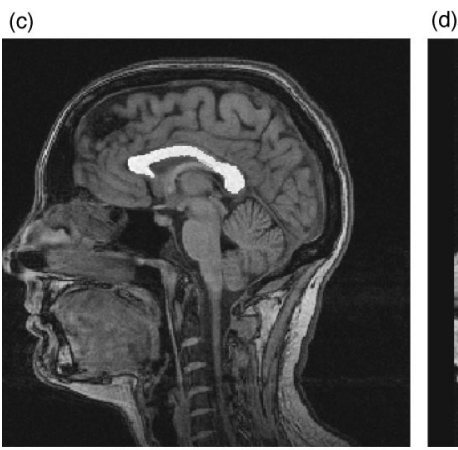

(d)

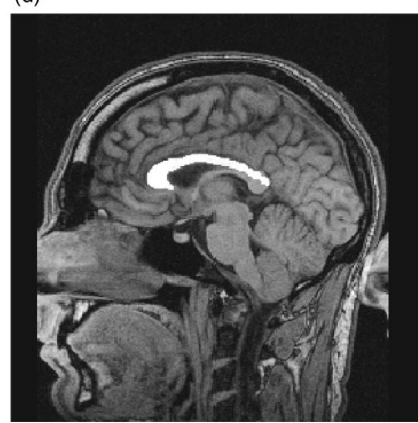

Fig. 16 Fornix removal by extending a line posteriorly from $A$.
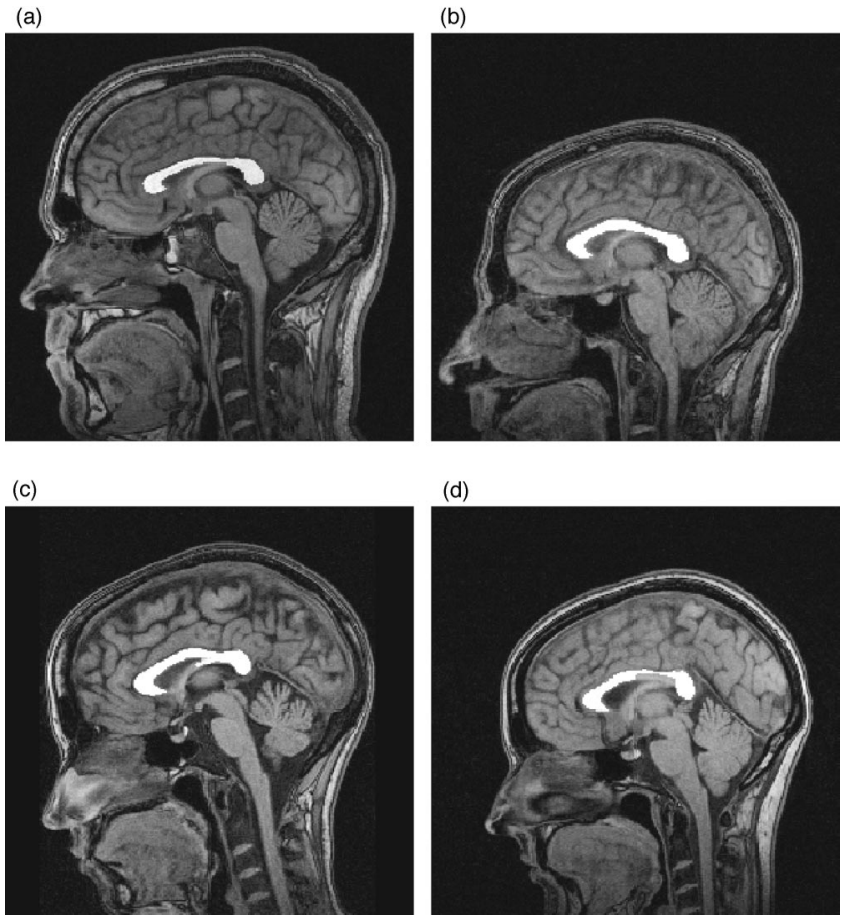

Fig. 17 Fornix removal by applying a mask obtained from an adjacent image.

results where the fornix was removed by extending a line posteriorly from $A$. Although we obtained satisfactory results in 18 out of 22 images that included the fornix (Fig. $16 \mathrm{a}-\mathrm{c})$, the method did not work if the thickness posteriorly from $B$ is greater than the thickness at $A$ as can be seen in Fig. 16d. Fig. 17a-b shows some of the results where the fornix was removed using a mask. In this method, the fornix might not be completely removed (Fig. 17c) or some of the corpus callosum might be removed (Fig. 17d) if the location of the adjacent corpus callosum does not match exactly. However, finding the true boundary by removing the fornix may be problematic in some sense since the gray levels are almost identical (Fig. 15). Therefore, if the fornix has to be removed to find the true boundary, the process needs to be guided by an expert in brain imaging.

\section{Experiments and Results}

We applied the proposed procedure to find the corpus callosum in midsagittal brain MR images ( 256 by 256, 256 gray levels). The proposed algorithm was tested on 120 subjects and we obtained generally very satisfactory results. Figs. 18-20 show the results with the corpus callosum found by the proposed algorithm highlighted. It is noted that the results are shown in Figs. 18-20 just because we have too many images. As can be seen, there are large variations in the location, size, orientation, and brightness. For instance, there are large variations in head area, from large (Figs. 18h, 18u, 18F) to small (Figs. 18n, 19L, 19M, 20o). Some of the subjects tilted forward (Figs. 18h, 18A), some tilted backward (Figs. 19H, 20j, 20v). In some images, artifacts from neighboring slices are included (Figs. $18 \mathrm{u}, 18 \mathrm{~J}$ ). Some images (Figs. 18D, 19o) are relatively bright and some are dark (Figs. 18i, 18O, 19M). In fact, the average gray level of the head area of Fig. 19o is about 114 
(a)

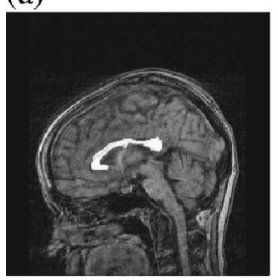

(g)

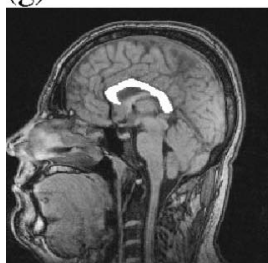

(m)

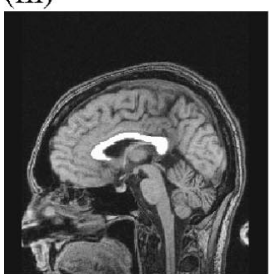

(s)

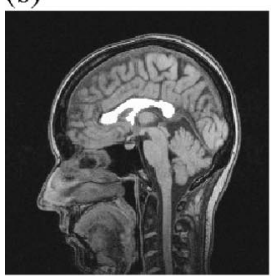

(y)

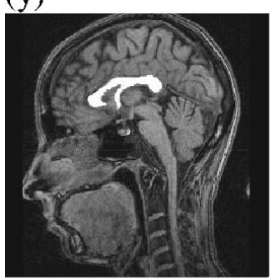

(E)

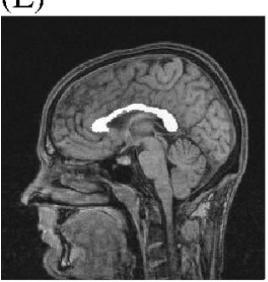

(K)

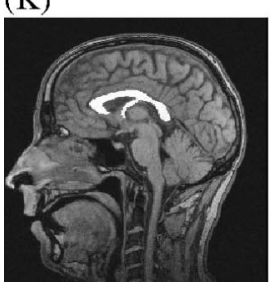

(b)

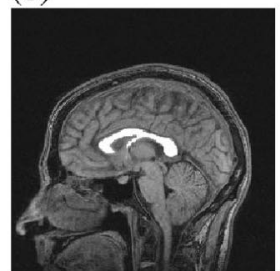

(h)

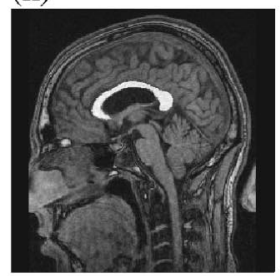

(n)

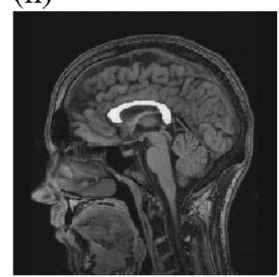

(t)

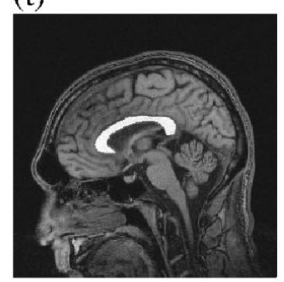

(z)

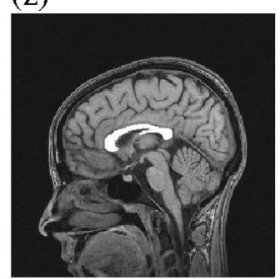

(F)

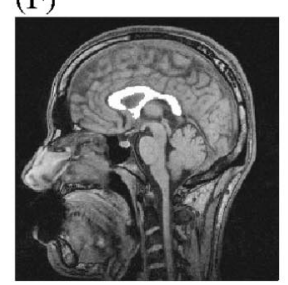

(L)

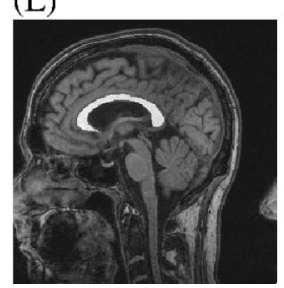

(c)

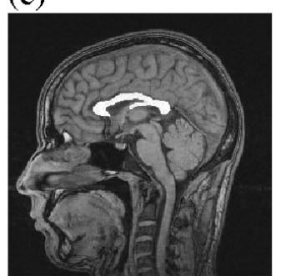

(i)

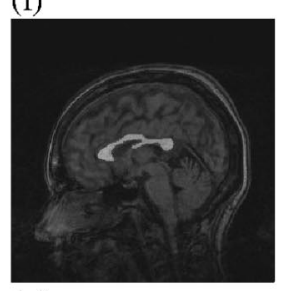

(o)

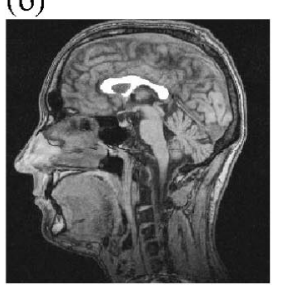

(u)

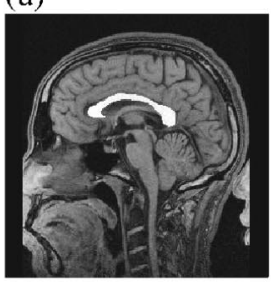

(A)

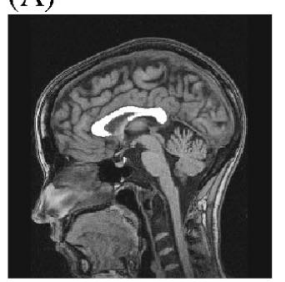

(G)

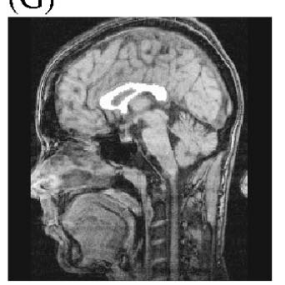

(M)

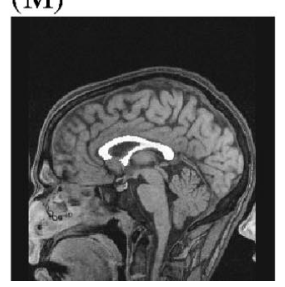

(d)

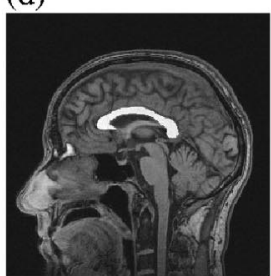

(j)

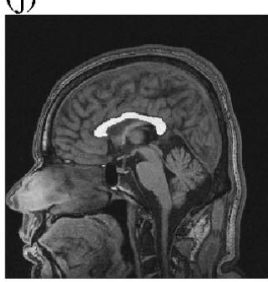

(p)

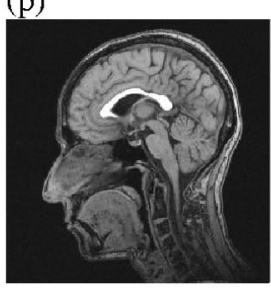

(v)

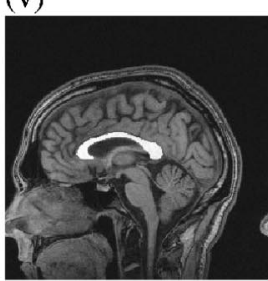

(B)

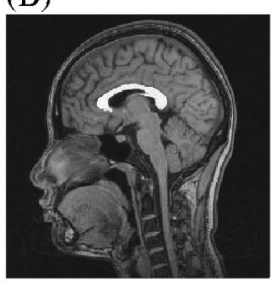

(H)

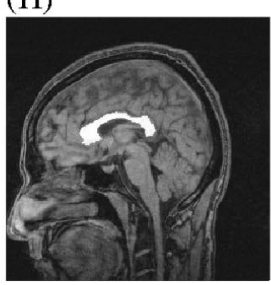

(N)

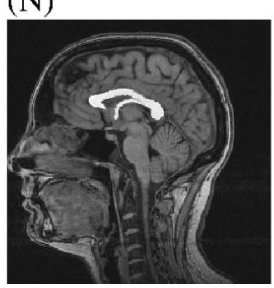

(e)

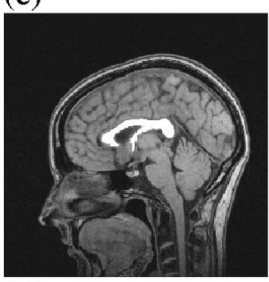

(k)

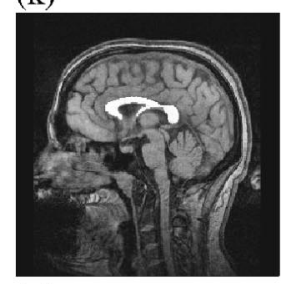

(q)

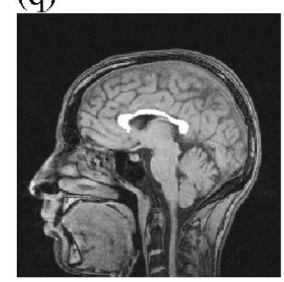

(w)

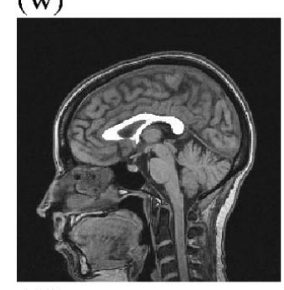

(C)

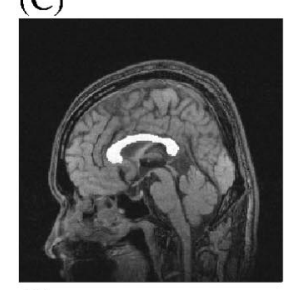

(I)

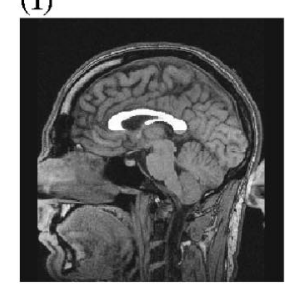

(O)

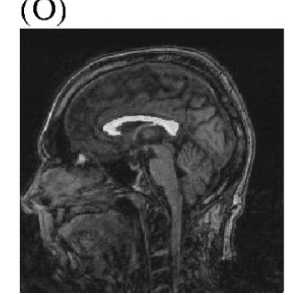

(f)

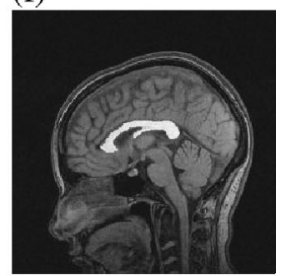

(1)

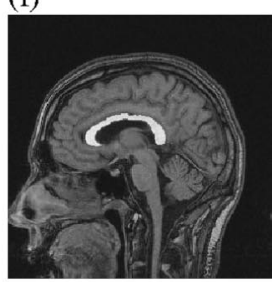

(r)

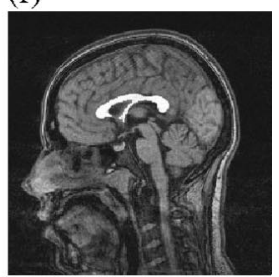

(x)

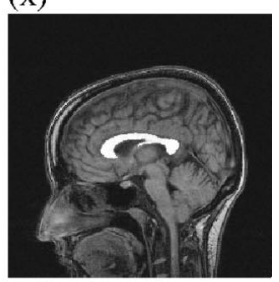

(D)

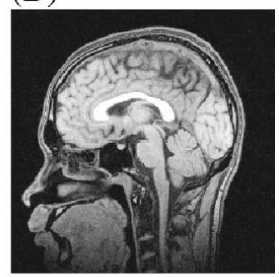

(J)

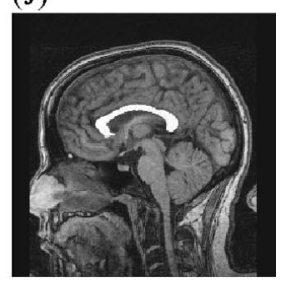

(P)

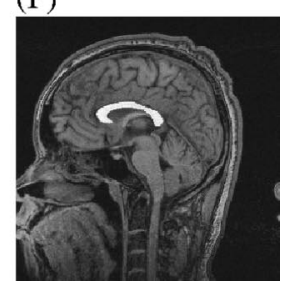

Fig. 18 The results obtained by the proposed algorithm.

while it is about 38 in Fig. 18i. Out of 120 images, the proposed algorithm was able to find the corpus callosum reasonably accurately. However, in 3 images, the corpus callosum found by the proposed algorithm was not perfect
(Figs. 20H-J). In Fig. 20H, a part of the corpus callosum is missing and Fig. 20I includes an artifact. In Fig. 20J, the proposed algorithm did not find the corpus callosum at all. A close examination reveals that the images were not the 
(a)

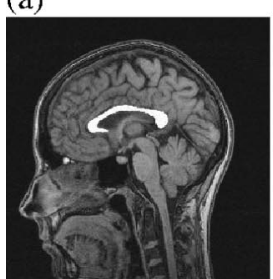

(g)

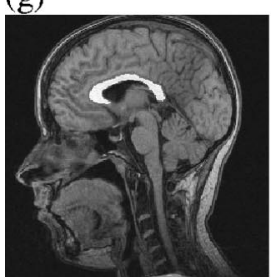

(m)

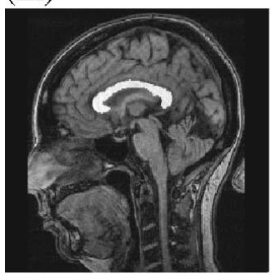

(s)

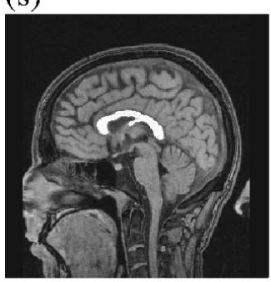

(y)

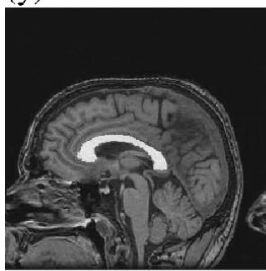

(E)

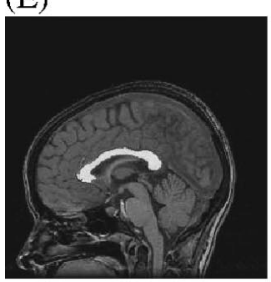

(K)

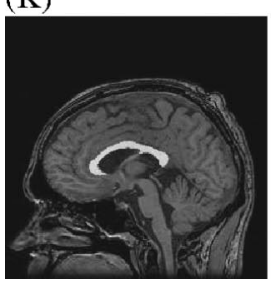

(b)

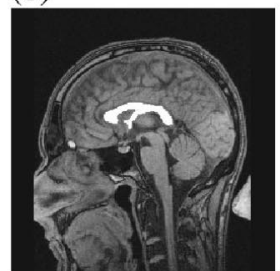

(h)

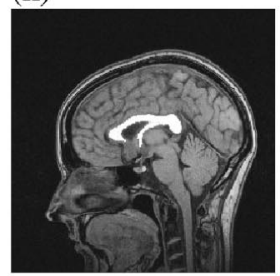

(n)

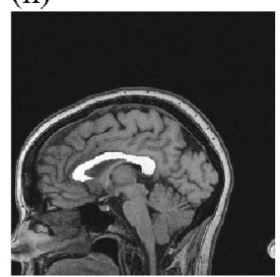

(t)

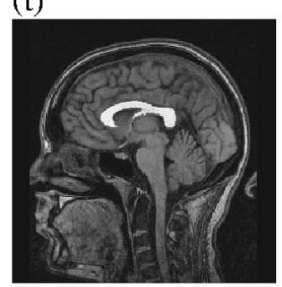

(z)

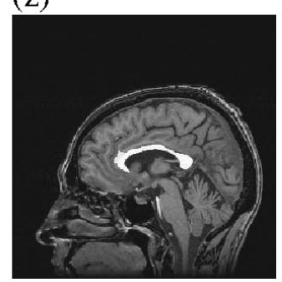

(F)

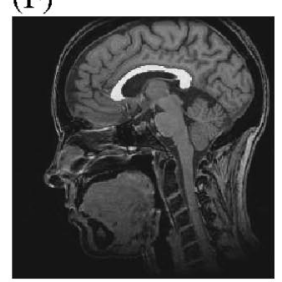

(L)

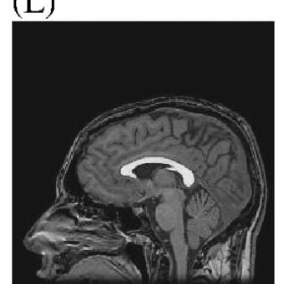

(c)

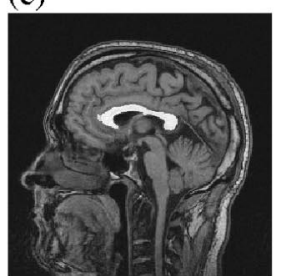

(i)

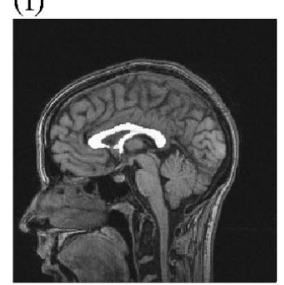

(o)

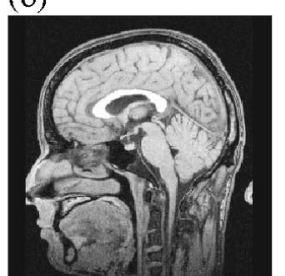

(u)

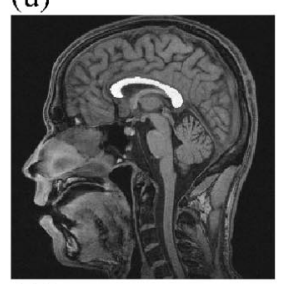

(A)

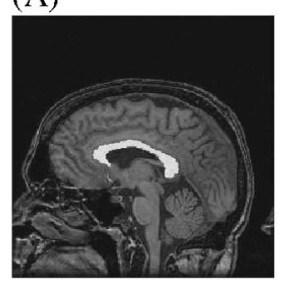

(G)

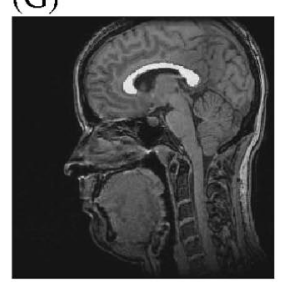

(M)

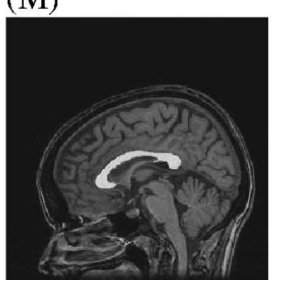

(d)

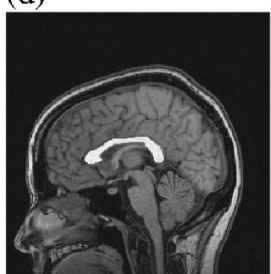

(j)

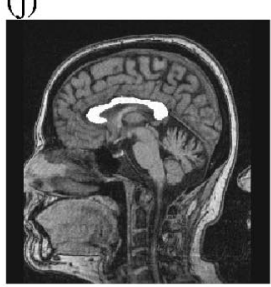

(p)

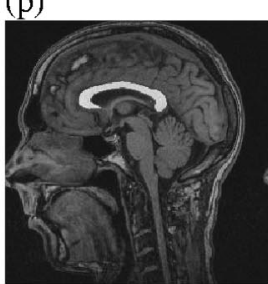

(v)

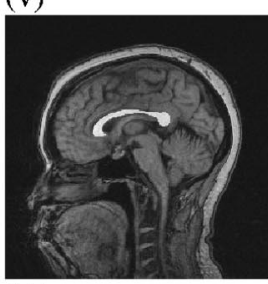

(B)

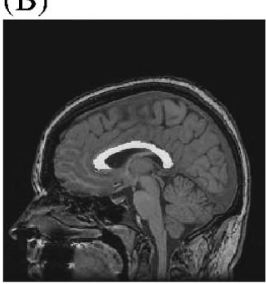

(H)

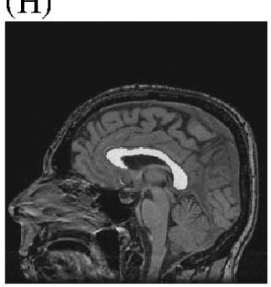

(N)

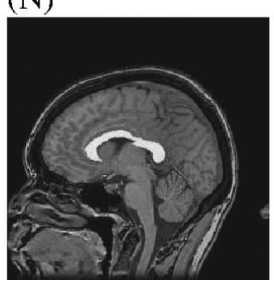

(e)

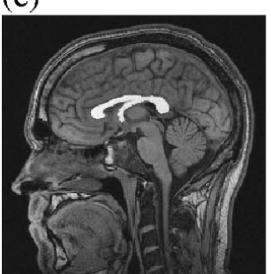

(k)

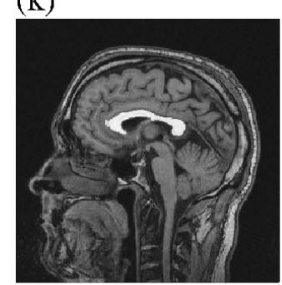

(q)

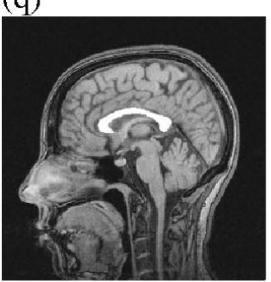

(w)

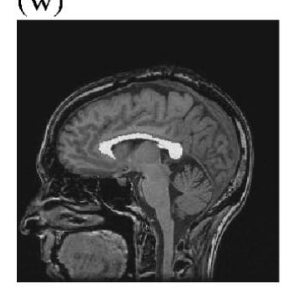

(C)

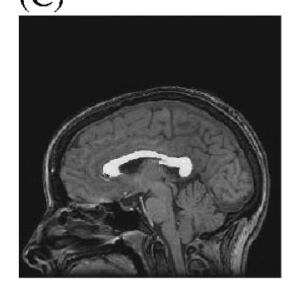

(I)

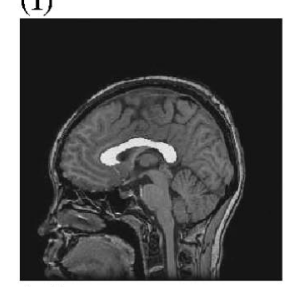

(O)

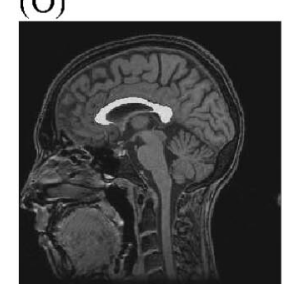

(f)

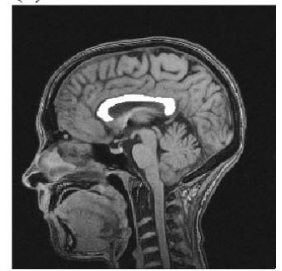

(1)

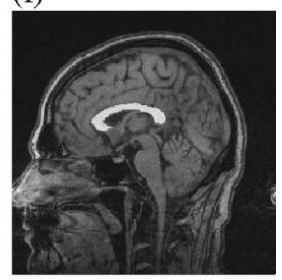

(r)

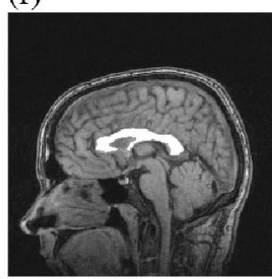

(x)

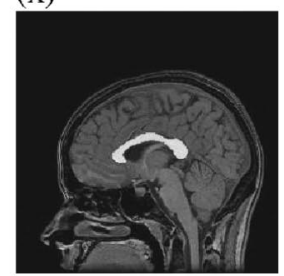

(D)

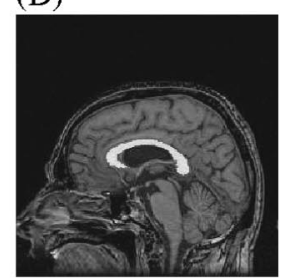

(J)

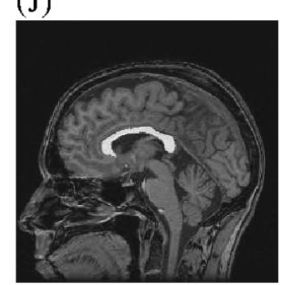

(P)

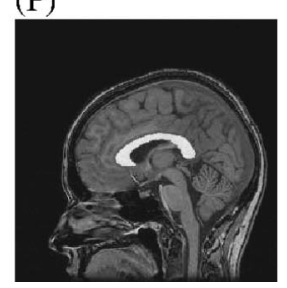

Fig. 19 The results obtained by the proposed algorithm.

midsagittal images. If the images came from the midsagittal images, better results would be obtained. Also, the head position of Fig. 20J was very different from the normal position. Although the results are generally satisfactory, ad- ditional processing may be required in some subjects to remove the fornix depending on applications. Out of 117 images where the corpus callosum was reasonably accurately segmented, 22 images also included the fornix. The 
(a)

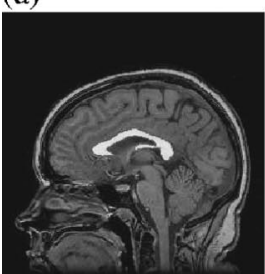

(g)

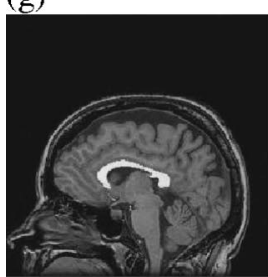

(m)

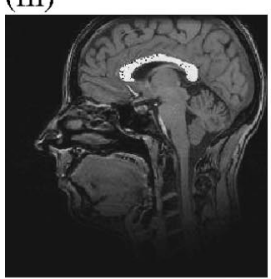

(s)

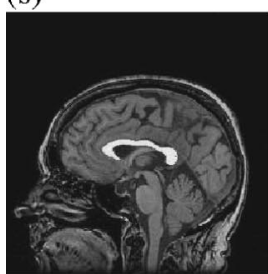

(y)

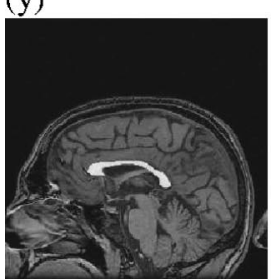

(E)

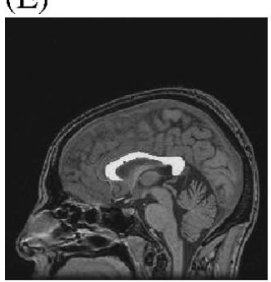

(b)

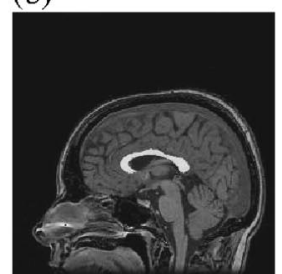

(h)

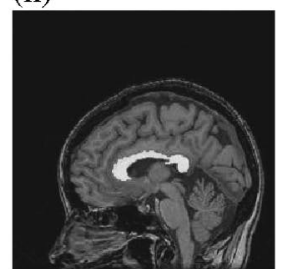

(n)

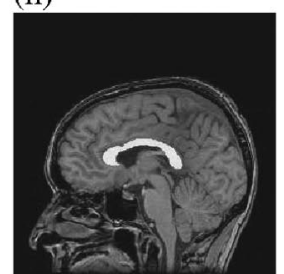

(t)

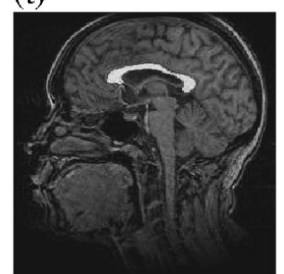

(z)

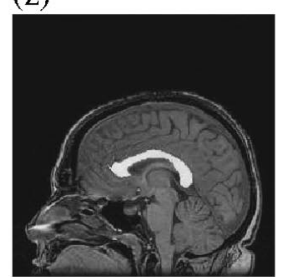

(F)

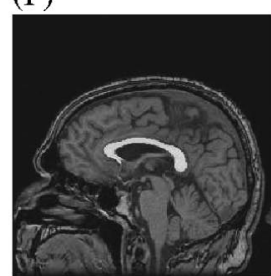

(c)

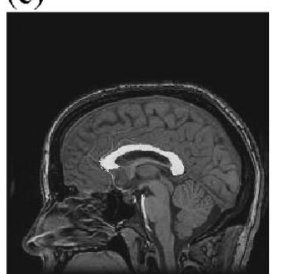

(i)

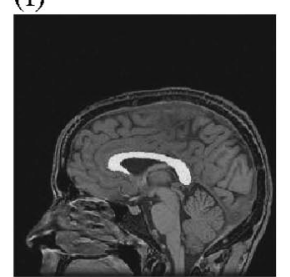

(o)

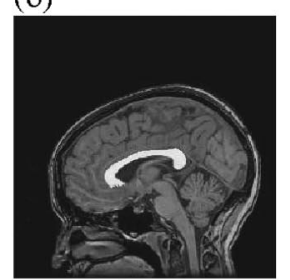

(u)

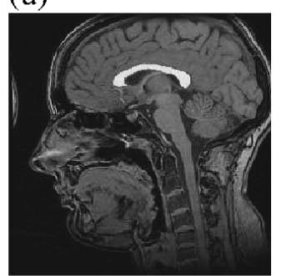

(A)

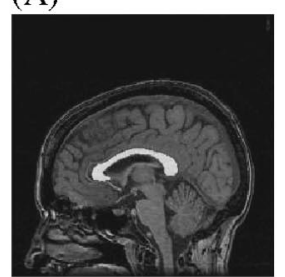

(G)

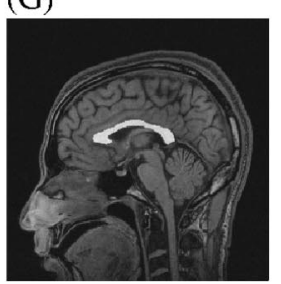

(d)

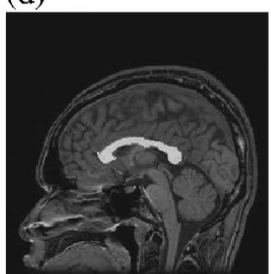

(j)

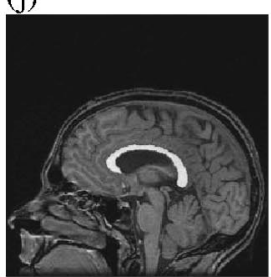

(p)

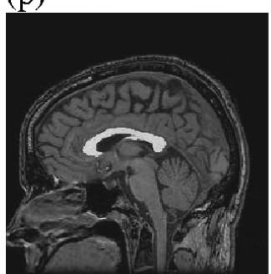

(v)

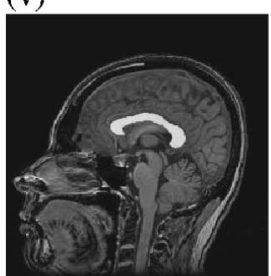

(B)

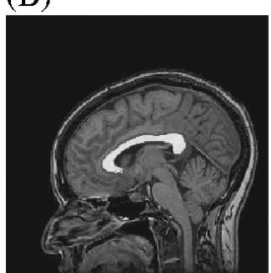

(H)

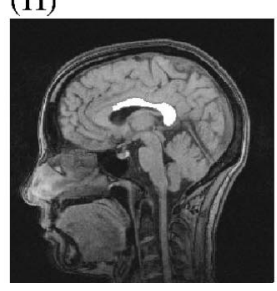

(e)

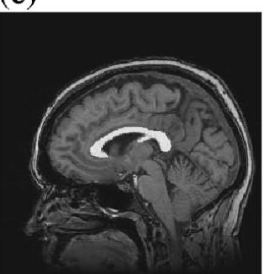

(k)

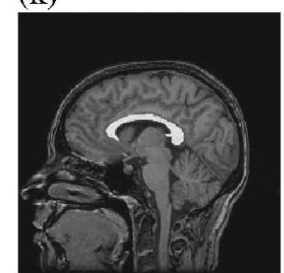

(q)

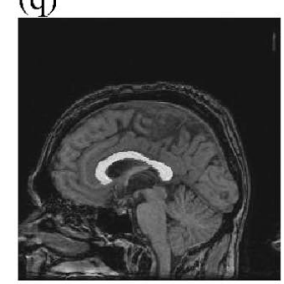

(w)

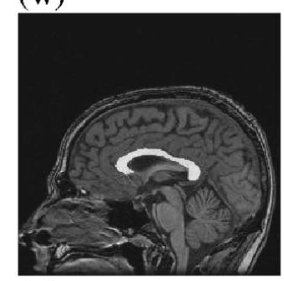

(C)

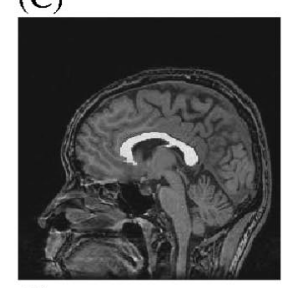

(I)

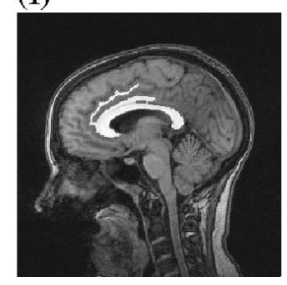

(f)

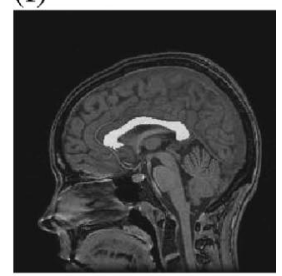

(1)

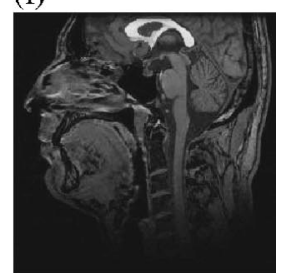

(r)

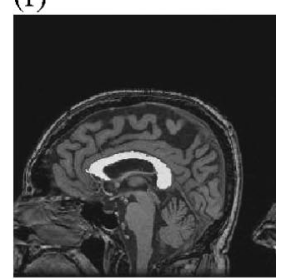

(x)

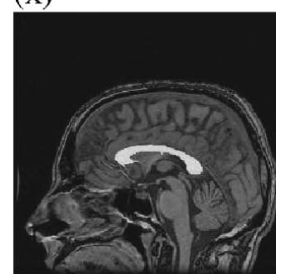

(D)

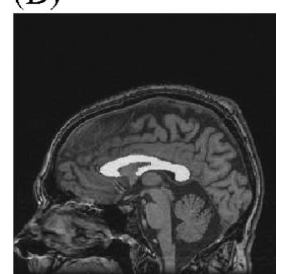

(J)

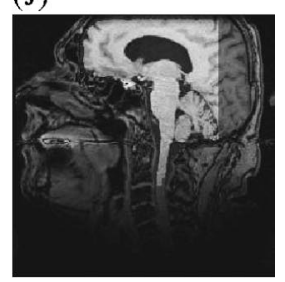

Fig. 20 The results obtained by the proposed algorithm.

fornix was reasonably accurately separated in 18 of these by extending a line posteriorly from $A$ (Fig. 16).

\section{Conclusion}

We propose an automatic algorithm that segments the corpus callosum from midsagittal brain MR images. The algorithm utilizes thresholding and region-based matching by directed window region growing. The boundary between the corpus callosum and surrounding tissues can be difficult to detect and, in some cases, artifacts are included in the segmented corpus callosum. To remove such artifacts, a new border path pruning method is proposed. Experiments showed that the technique can be successfully applied to a wide range of MR images. Once the corpus callosum is segmented from surrounding tissues, it can be used as a reliable landmark to find other brain structures, help segment the brain from surrounding tissues, and register images across individuals by matching the internal cerebral structures. Extension of the described algorithm into three dimensions should facilitate volumetric determination of the size of the corpus callosum, segmentation of cerebral structures, and possibly even coregistration of MR and PET 
images, as the corpus callosum has low and cortical regions and high rates of metabolism and blood flow in PET images.

\section{References}

1. N. R. Pal and S. K. Pal, "A review on image segmentation techniques," Pattern Recogn. 26(9), 1277-1294 (1993).

2. R. M. Haralick and L. G. Shapiro, "Survey, image segmentation techniques," Comput. Vis. Graph. Image Process. 29, 100-132 (1985).

3. R. Dekerck, C. Cornelis, and M. Bister, "Segmentation of medical images," Image Vis. Comput. 11(8), 486-503 (Oct. 1993).

4. T. Pun, "Entropic thresholding, a new approach," Comput. Graph. Image Process. 16, 210-239 (1981).

5. P. K. Sahoo, S. Soltani, A. K. C. Wong, and Y. C. Chen, "A survey of thresholding techniques," Comput. Vis. Graph. Image Process. 41 233-260 (1988)

6. J. F. Canny, "A computational approach to edge detection," IEEE Trans. Pattern. Anal. Mach. Intell. 8, 679-698 (1986).

7. J. Lin, K. Cheng, and C. Mao, "A fuzzy Hopfield neural network for medical image segmentation," IEEE Trans. Nucl. Sci. 43(4), 23892398 (1996).

8. X. Yu and J. Yla-Jaaski, "Interactive surface segmentation for medical images," Proc. SPIE 2564, 519-527 (1995).

9. A. Yezzi, S. Kichenassamy, A. Kumar, P. Olver, and A. Tannenbaum, "A geometric snake model for segmentation of medical imagery," IEEE Trans. Med. Imaging 16(2), 199-209 (1997).

10. D. Koechner, H. Petropoulos, R. P. Eaton, B. L. Hart, and W. M. Brooks, "Segmentation of small structures in MR images: Semiautomated tissue hydration measurement," JMRI 5(3), 347-351 (1995).

11. M. Bomans, K. H. Hohne, U. Tiede, and M. Riemer, "3-D segmentation of MR images of the head for 3-D display,"' IEEE Trans. Med. Imaging 9(2), 177-183 (1990)

12. C. Tsai, B. S. Manjunath, and R. Jagadeesan, "Automated segmentation of brain MR images," Pattern Recogn. 28(12), 1825-1837 (1995).

13. R. Deklerck, J. Cornelis, and M. Bister, "Segmentation of medical images," Image Vis. Comput. 11(8), 486-503 (1993).

14. N. Shareef, D. Wang, and R. Yagel, "Segmentation of medical images using LEGION," IEEE Trans. Med. Imaging 18(1), 74-91 (1999).

15. A. Chakraborty and J. Duncan, "Game-theoretic integration for image segmentation," IEEE Trans. Pattern. Anal. Mach. Intell. 21(1), 12-30 (1999).

16. M. Worring, A. Smeulders, L. Staib, and J. Duncan, "Parameterized feasible boundaries in gradient vector fields," Comput. Vis. Image Underst. 63(1), 135-144 (1996)

17. L. K. Arata, A. P. Dhawan, J. P. Broderick, M. F. Gaskil-Shipley, A. V. Levy, and N. D. Volkow, "Three-dimensional anatomical modelbased segmentation of MR brain images through principal axes registration," IEEE Trans. Biomed. Eng. 42(11), 1069-1078 (1995).

18. S. Chaudhuri, S. Chatterjee, N. Katz, M. Nelson, and M. Goldbaum, "Detection of blood vessels in retinal images using two-dimensional matched filters," IEEE Trans. Med. Imaging 8(3), 263-269 (1989).

19. M. C. Clark, L. O. Hall, D. B. Goldgof, R. Velthuizen, F. R. Murtagh, and M. S. Silbiger, "Automatic tumor segmentation using knowledge-based techniques," IEEE Trans. Med. Imaging 17(2), 187-201 (1998).

20. J. W. Klingler, Jr., B. L. Vaughan, T. D. Fraker, and L. T. Andrews, Jr., "Segmentation of echocardiographic images using mathematical morphology,"' IEEE Trans. Biomed. Eng. 35(11), 925-934 (1988).

21. C. H. Chu, E. J. Delp, and A. J. Buda, "Detecting left ventricular endocardial and epicardial boundaries by digital two-dimensional echocardiography," IEEE Trans. Med. Imaging 7(2), 81-90 (1988).

22. A. Goshtasby and D. A. Turner, "Segmentation of cardiac cine MR images for extraction of right and left ventricular chambers," IEEE Trans. Med. Imaging 14(1), 56-64 (1995).

23. B. A. Georgy, J. Hesselink, and T. L. Jernigan, "MR imaging of the corpus callosum," AJR 160, 949-955 (1993).

24. M. F. Casanova, R. D. Sanders, T. E. Goldberg, L. B. Bigelow, G. Christison, E. F. Torrey, and D. R. Weinberger, "Morphometry of the corpus callosum in monozygotic twins discordant for schizophrenia: a magnetic resonance imaging study," J. Neurol. Neurosurg. Psychiatry 53, 416-421 (1990)

25. M. F. Casanova, M. Zito, T. Goldberg, A. Abi-Dargham, R. Sanders, L. B. Bigelow, D. F. Torrey, and D. R. Weinberger, "Shape distortion of the corpus callosum of monozygotic twins discordant for schizophrenia," Schizophr. Res. 3, 155-156 (1990).

26. M. F. Casanova, M. Zito, T. Goldberg, R. L. Suddath, E. F. Torrey, L. B. Bigelow, R. D. Sanders, and D. R. Weinberger, "Corpus callosum curvature in schizophrenic twins," Biol. Psychiatry 28, 83-84 (1990).

27. C. Colombo, A. Bonfanti, S. Livian, M. Abbruzzese, and S. Scarone, "Size of the corpus callosum and auditory comprehension in schizophrenics and normal controls,"' Schizophr. Res. 11, 63-70 (1993).

28. W. Gunther, R. Petsch, R. Steinberg, E. Moser, P. Streck, H. Heller, G. Kurtz, and H. Hippius, "Brain dysfunction during motor activation and corpus callosum alterations in schizophrenia measured by cerebral blood flow and magnetic resonance imaging," Biol. Psychiatry 29, 535-555 (1991).

29. L. Jacobsen, J. N. Giedd, J. C. Rajapakse, S. D. Hamburger, A. C. Vaituzis, J. A. Frazier, M. C. Lenane, and J. L. Rapoport, "Quantitative magnetic resonance imaging of the corpus callosum in childhood onset schizophrenia," Psychiatry Res. 68, 77-86 (1997).

30. P. Tibbo, P. Nopoulos, S. Arndt S, and N. C. Andreasen, "Corpus callosum shape and size in male patients with schizophrenia," Biol. Psychiatry 44, 405-412 (1998).

31. M. M. Husain, G. S. Figiel, S. N. Lurie, O. B. Boyko, Jr., E. H. Ellinwood, C. B. Nemeroff, and K. R. Krishnan, "MRI of corpus callosum and septum pellucidum in depression," Biol. Psychiatry $\mathbf{2 9}$, 300-301 (1991)

32. M. Uematsu and H. Kaiya, "The morphology of the corpus callosum in schizophrenia, An MRI study," Schizophr. Res. 1, 391-398 (1988).

33. A. Rossi, P. Stratta, M. Callucci, I. Amicarelli, R. Passariello, and M. Casacchia, "Standarized magnetic resonance image intensity study in schizophrenia," Psychiatry Res. 25, 223-231 (1988).

34. P. W. R. Woodruff, I. C. McManus, and A. S. David, "Meta-analysis of corpus callosum size in schizophrenia," J. Neurol., Neurosurg. Psychiatry 58, 457-461 (1995)

35. J. A. Coffman, R. A. Bornstein, and S. C. Olson, "Cognitive impairment and cerebral structure by MRI in bipolar disorder," Biol. Psychiatry 27, 1188-1196 (1990).

36. J. C. Wu, M. S. Buchsbaum, J. C. Johnson, T. G. Hershey, F. A. Wagner, C. Teng, and S. Lottenberg, "Magnetic resonance and positron emission tomography imaging of the corpus callosum: size, shape and metabolic rate in unipolar depression," J. Affect Disord. 28, 15-25 (1993).

37. P. Hauser, I. D. Dauphinais, W. Berrettini, L. E. DeLisi, J. Gelernter, and R. M. Post, "Corpus callosum dimensions measured by magnetic resonance imaging in bipolar affective disorder and schizophrenia,' Biol. Psychiatry 26, 659-658 (1989).

38. L. H. Staib and J. S. Duncan, "Boundary finding with parametrically deformable models," IEEE Trans. Patt. Recog. Mach. Intel. 14(11), 1061-1075 (1992).

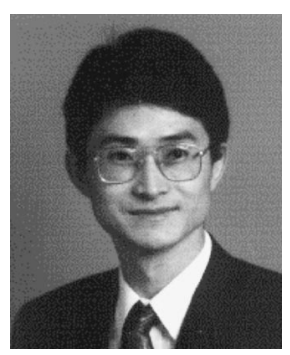

Chulhee Lee received the BS and MS degrees in electronics engineering from Seoul National University in 1984 and 1986, respectively, and the PhD degree in electrical engineering from Purdue University, West Lafayette, Indiana, in 1992 From 1986 to 1987 , he was a researcher in the Acoustic Laboratory at Technical University of Denmark (DTU). From 1993 to 1996, he worked with National Institutes of Health, Bethesda, Maryland. In 1996, he joined the faculty of the Department of Electronic Engineering, Yonsei University, Seoul, Korea. His research interests include image/ signal processing, pattern recognition, and neural networks. Dr. Lee is a member of Tau Beta Pi, Eta Kappa Nu, and KSEA.

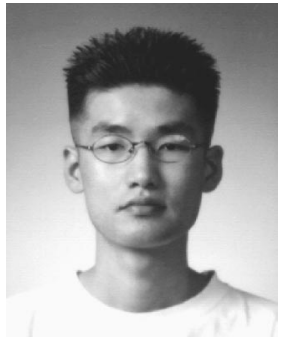

Shin Huh received the BS degree in electronics engineering from Yonsei University in 1998. He is currently working towards the MS degree in electronics engineering at Yonsei University. His current research interests include image segmentation and image/signal processing. 
Lee et al.: Automated segmentation of the corpus callosum in midsagittal ...

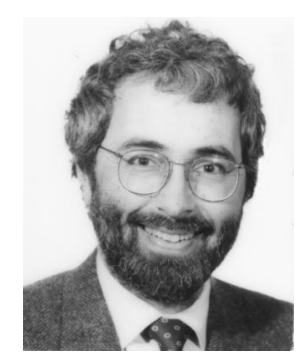

Terence A. Ketter obtained his medical degree from the University of Toronto and had internship and residency training in psychiatry at the University of California at San Francisco. He had subsequent fellowship training in psychopharmacology and brain imaging research methods in the Biological Psychiatry Branch of the National Institute of Mental Health in Bethesda, Maryland. He is currently associate professor of Psychiatry \& Behavioral Sciences and chief of the Bipolar Disorders Clinic at the Stanford University School of Medicine in Stanford, California. Dr. Ketter's research interests include the use of brain imaging methods such as positron emission tomography (PET) and magnetic resonance imaging (MRI) and magnetic resonance spectroscopy (MRS) to better understand the neurobiology of mood (and especially bipolar) disorders. Dr. Ketter has also investigated the use of novel therapies such as new anticonvulsant drugs and combinations of medications in the treatment of mood disorders. His current work includes exploring the possibility of using brain imaging techniques to more effectively target treatments in mood disorders.

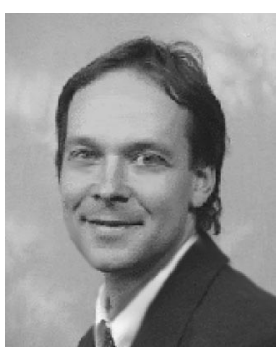

Michael Unser received the MS (summa cum laude) and $\mathrm{PhD}$ degrees in electrical engineering in 1981 and 1984, respectively, from the Swiss Federal Institute of Technology (EPFL) in Lausanne, Switzerland. From 1985 to 1997, he was with the Biomedical Engineering and Instrumentation Program, National Institutes of Health Bethesda, USA, where he was heading the Image Processing Group. He is now professor and head of the Biomedical Imaging Group at the EPFL. His main research area is biomedical image processing. He has a strong interest in sampling theories, multiresolution algorithms, wavelets, and the use of splines for image processing. $\mathrm{He}$ is the author of over 80 published journal papers in these areas. Dr. Unser is an associate editor for the IEEE Transactions on Medical Imaging; he is also on the editorial boards of Signal Processing, the Journal of Visual Communication and Image Representation, and Pattern Recognition. He is a member of the Image and Multidimensional Signal Processing Committee of the IEEE Signal Processing Society and was a former associate editor for the IEEE Transactions on Image Processing (1992-1995) and the IEEE Signal Processing Letters (1994-1998). He co-organized the 1994 IEEE-EMBS Workshop on Wavelets in Medicine and Biology, and serves as conference chair for SPIE's Wavelet Applications in Signal and Image Processing, which has been held annually since 1993. He received the Dommer prize for excellence from the Swiss Federal Institute of Technology in 1981, the research prize of the Brown-Boveri Corporation (Switzerland) for his thesis in 1984, and the IEEE Signal Processing Society's 1995 Best Paper Award. In January 1999, he was elected Fellow of the IEEE with the citation: "for contributions to the theory and practice of splines in signal processing." 